\title{
A Simplified Method of Measuring the Marginal Powers of Spectacle Lenses ${ }^{1}$
}

\author{
Francis E. Washer
}

\begin{abstract}
A device is described that permits the measurement of marginal meridional power of spectacle lenses on a standard commercially available vertex power measuring instrument. The device consists of a hemispherical mounting surface and a variable prism which when mounted on a standard instrument permits the measurement of meridional power with respect to the vertex sphere in the extra-axial regions. It is used primarily to measure the meridional powers in the region of the lens normally used at a viewing angle of $30^{\circ}$ from the axis. Some measurements on typical lenses are reported.
\end{abstract}

\section{Introduction}

In the course of an investigation of the properties of "corrected curve" spectacle lenses with the aim of preparing performance specifications for use by the Veterans' Administration, it became evident that new test methods were necessary in order to measure the power of spectacle lenses in the extra-axial region. In another paper, ${ }^{2}$ a new instrument was described that is capable of making all of the necessary measurements. This new instrument is essentially an absolute instrument and is somewhat too complex to be well suited to general use.

A method has therefore been developed whereby standard vertex power measuring instruments of the type ordinarily found in opticians' laboratories can be modified to permit the measurement of the degree of marginal correction of spectacle lenses. The modifications consist of a few parts that can readily be attached to the usual type of instrument such as the "Vertometer", "Lensometer", or similar instruments used in measuring vertex power of spectacle lenses.

Comparison of measurements of marginal power made on a number of spectacle lenses (1) using such a modified instrument and (2) using the spectacle tester, shows close agreement. While not as versatile as the spectacle tester, a modified lense measuring instrument can be used for the rapid checking of lenses for a selected extra-axial region. It will generally be more practical for many workers to employ such a modification on an existing instrument than attempt to make a device like the precision. spectacle tester.

The devices described in the present paper were designed for use on a vertometer belonging to this laboratory. It must be emphasized that the same modification can be used on a lensometer or similar commercially available type of instrument. It is probable that slight changes may be necessary depending upon the type of instrument, but the fundamental principles will remain the same.

1 This work was performed in connection with a research project sponsored by Veterans' Administration.

2 F. E. Washer, J. Opt. Soc. Am. (in press).

\section{Theory}

In prescribing spectacle lenses, it is customary to specify the lenses in terms of spherical and cylindrical power as measured on the optical axis. It is presumed that this prescribed power represents the best estimate of the correction required by the potential user. In looking through the spectacle lens only a relatively small area is used at a time and the degree of correction afforded when looking through the axial area is as prescribed. It is of interest to know if this same degree of correction prevails as the eye rolls and extra-axial areas are brought into use. The problem is not simple. Viewing conditions for the rolling eye differ markedly from those that prevail for the stationary eye looking through the axial region of the lens. The distance separating the rear surface of the spectacle lens and front surface of the cornea changes continuously as the eye rolls with the separation usually being a minimum in the axial region. In addition, the light incident on the front surface of the lens is subjected to refracting conditions which differ with the angular separation of the used area from the axial area of the lens.

These difficulties can be circumvented by making all refracting power measurement with respect to the vertex sphere. The vertex sphere has its center at the center of rotation. of the eye and its surface is tangent to the rear surface of the spectacle lens at the point of intersection of the optical axis of the spectacle lens and its rear surface. The radius of the vertex sphere is $27.0 \mathrm{~mm}$. By referring all measurements to the vertex sphere, comparisons can be readily made between refracting power at any specified extra-axial region and that at the optical axis.

In attempting to measure the refractive powers of a spectacle lens in the marginal regions as well as in the axial region, considerable confusion results in the use of the terms "spherical" and "cylindrical" power. In this paper, therefore, principal emphasis is placed upon the "meridional power". Figure 1 shows the region of the lens for which the values of the refractive powers are determined. The task of comparing marginal and axial power is simplified by regarding the several regions of the lens under test as separate lenses, designated $O, A$, and $B$ in figure 2 . 


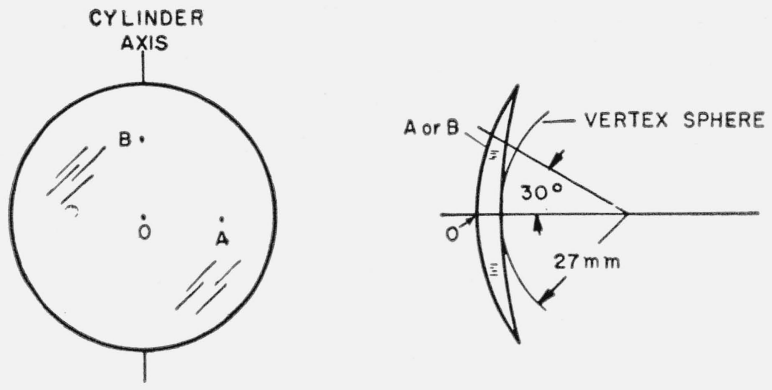

FIGURE 1. Schematic diagram showing the regions of principal interest and the vertex sphere.

The optic axis is indicated at $\mathrm{O}$. The marginal power with respect to the vertex sphere is measured at points $A$ and $B$.

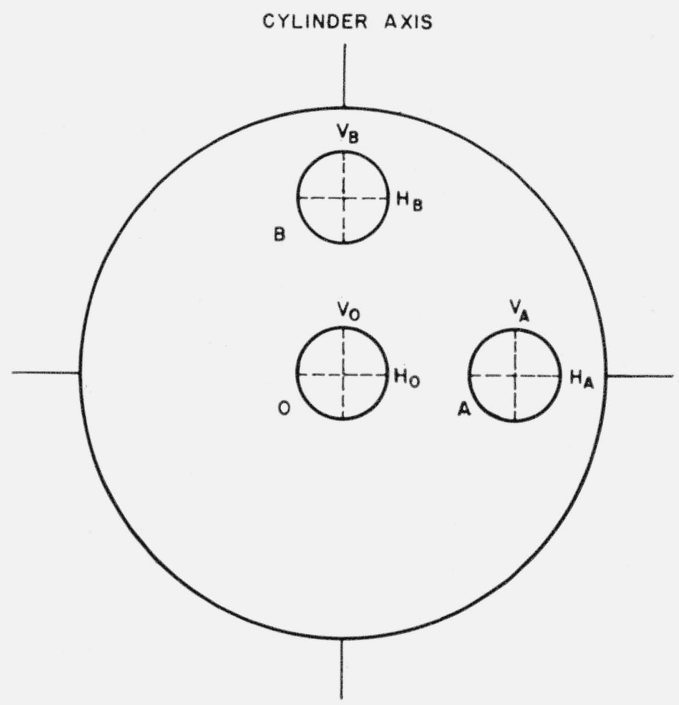

FIgURE 2. Schematic diagram showing the actual regions where measurements are made.

Each small area of the spectacle lens is treated as a separate lens and comparisons are made of the respective powers. The meridional axes of the various areas are as indicated.

The various meridians are also indicated in the figure.

\subsection{Definitions}

For convenience, the definitions of all symbols used in describing the various powers and their interrelations are collected into this section. This system specifying the powers in various areas is patterned to some extent after the system devised by Whitwell and referred to by Emsley and Swaine. ${ }^{3}$

$V_{P}=$ One of the principal meridional powers. It is given by the sum of the prescribed spherical and prescribed cylindrical power. It is the maximum meridional power when the prescribed powers are positive.

$H_{P}=$ The second principal meridional power. It is usually the same as the spherical power. It is the minimum meridional power when the prescribed powers are positive.

$C_{P}=$ Prescribed cylindrical power. It is the difference between the principal meridional powers and is given by the relation $C_{P}=V_{P}-H_{P}$.

${ }^{3}$ H. H. Emsley and Wm. Swaine, Ophthalmic Lenses, 6th ed. (Hatton Press Ltd., London, 1951).
$V_{O}=$ Measured value of one of the meridional powers at the optical center of the lens. It is the measured power for vertical lines.

$H_{O}=$ Measured value of the second meridional power at the optical center of the lens. It is the measured power for horizontal lines. (In the absence of cylindrical power, $V_{O}=H_{O}$.)

$C_{O}=$ The measured cylindrical power at the optical center of the lens. It is obtained from the relation, $C_{O}=V_{O}-H_{O}$.

$V_{A}, V_{B}=$ The measured marginal powers at points $A$ and $B$ in the same meridian as $V_{O}$ or parallel to the meridian of $V_{O}$. These are the measured marginal powers for vertical lines.

$H_{A}, H_{B}=$ The measured marginal powers at points $A$ and $B$ in the same meridian as $H_{O}$ or parallel to the meridian of $H_{O}$. These are the measured marginal powers for horizontal lines.

$C_{A}=$ The measured cylindrical power at point $A$, given by the relation $C_{A}=V_{A}-H_{A}$

$C_{B}=$ The measured cylindrical power at point $B$, given by the relation $C_{B}=V_{B}-H_{B}$.

\subsection{Additional Relations}

The following formulas are used in computing the departures of the measured values from the specified values for the axial region and the departures of the measured marginal values from the corresponding measured axial values.

$\begin{array}{llr}\Delta V_{O}=V_{O}-V_{P} & \Delta V_{A}=V_{A}-V_{O} & \Delta V_{B}=V_{B}-V_{O} \\ \Delta H_{O}=H_{O}-H_{P} & \Delta H_{A}=H_{A}-H_{O} & \Delta H_{B}=H_{B}-H_{O} \\ \Delta C_{O}=C_{O}-C_{P} & \Delta C_{A}=C_{A}-C_{O} & \Delta C_{B}=C_{B}-C_{O}\end{array}$

\section{Method of Measurement}

\subsection{Measurement of Axial Power}

The usual type of commercially available vertex power measuring instrument is designed primarily for use in the measurement of axial power. The diagrammatic sketch in figure 3 shows the position of a lens under test on a typical instrument. The back surface of the lens is held firmly against the fixed reference opening, $M$, of the instrument. The optical axis of the lens is centered with respect to the opening $M$. Light from the movable target and calibrated optical system (not shown) passes through
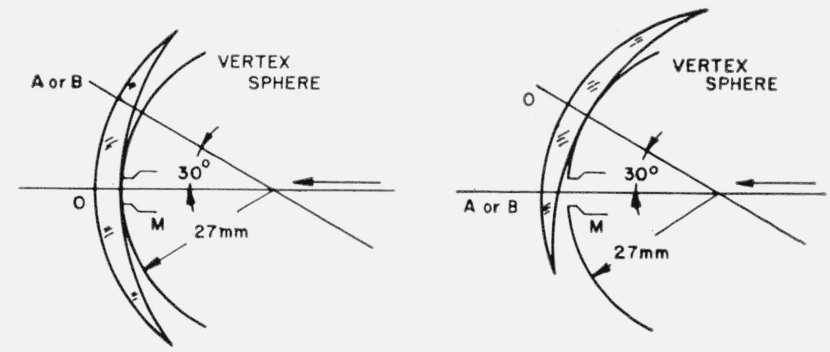

FIGURE 3. Schematic diagram showing the positions assumed by a spectacle lens when tested on a modified vertometer.

The left part of the figure shows arrangement for measurement of axial power. The fixed reference opening against which the lens is pressed is shown at $\mathbf{M}$ The optical center $O$ is made to coincide with the center of $M$. The trace of the vertex sphere is also shown. In the right part of the figure, point $O$ is pressed against the vertex sphere as shown. Light from $M$ passes through the lens under test at points A or B and the marginal power can thus be measured with respect to the vertex sphere. 
$M$ and is incident upon the back surface of the lens under test. The target is viewed through the front of the lens with the aid of a telescope. After position of best focus is found, the back vertex power of the spectacle lens is read from the scale of a movable drum calibrated in diopters.

\subsection{Measurement of Marginal Power}

It is possible to measure the marginal power of a spectacle lens with respect to the vertex sphere by appropriate modification of a standard instrument. One modification that has been developed in this laboratory and found satisfactory is the addition of a hemispherical seating surface against which the lens under test is held. The radius of this surface is $27 \mathrm{~mm}$ which is the value customarily accepted as being the axial distance separating the back surface of a spectacle lens and the center of rotation of the average eye. This surface is so positioned that the edge of the standard opening $M$, shown in figure 3 , is tangent to the hemispherical surface. For measurements of marginal power at $30^{\circ}$ from the axis, a dot is marked on the hemispherical surface to locate a point $30^{\circ}$ from the axis. The optical center of the lens during test is pressed against this dot as indicated in figure 3, which also shows the relative position of the marginal area $A$ or $B$ with respect to the opening $M$. Because of the prism power introduced by this decentration and tipping of the spectacle lens with respect to the opening $M$, it is necessary to use suitable prisms to bring the emergent light beam into the field of view of the observing telescope.

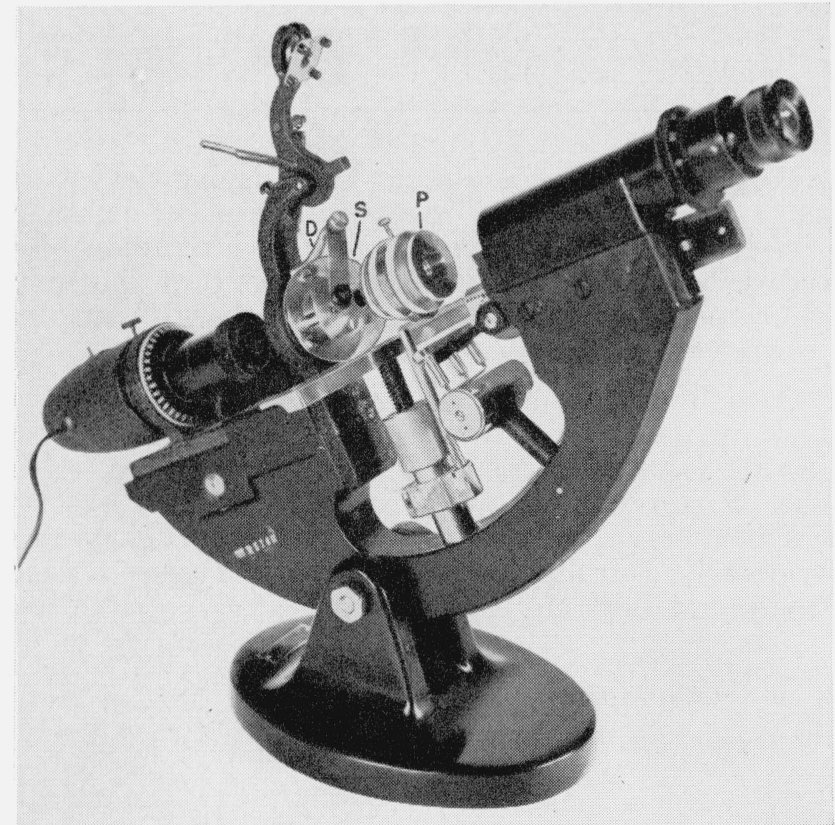

Figure 4. A vertometer modified for measuring marginal power. The hemispherical mounting surface is shown at $\mathrm{S}$; the clamping device is marked $\mathrm{D}$; and the variable prism is indicated by $\mathrm{P}$.

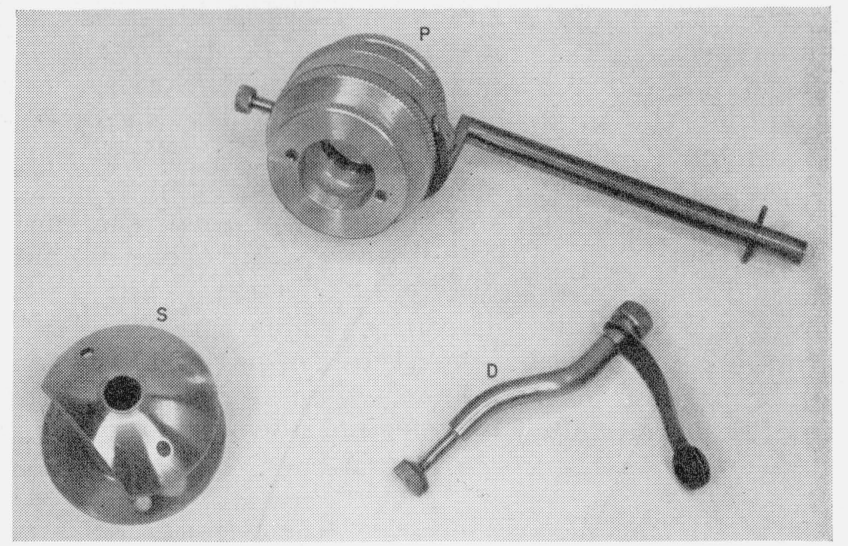

Figure 5. Principal parts of the auxiliary equipment used to modify a vertex power measuring instrument to permit measurement of marginal power.

The parts shown are the hemispherical surface, $\mathrm{S}$; the variable prism, P; and the clamping device, D.

An instrument equipped with these modifying devices is shown in figure 4 . The hemispherical surface is identified by the symbol $S$; the clamping device is marked $D$; and the variable prism in its holder is marked $P$. The principal parts of this auxiliary equipment are shown separately in figure 5 . The hemispherical attachment is so made that it fits over the regular mounting and does not prevent use of the instrument for the measurement of axial power. The prism holder contains two 15 diopter prisms that can rotate with respect to each other, thus permitting a variation in prism power ranging from 0 to 30 prism diopters. This range of prism power has been found adequate.

So long as unmounted lenses are being measured, this modified instrument can be used for the measurement of either axial or marginal power. It is not, however, practicable, in most instances, to use the modified instrument for the measurement of axial and marginal power for lenses mounted in spectacle frames. When measurements on such lenses are required, it will usually be necessary to remove the lenses from the frames prior to making the measurements.

\section{Results of Measurement}

The performance of several hundred lenses has been measured with this modified vertex power measuring instrument but for the purpose of this article, it is sufficient to show the results on a few lenses only in order to describe the measuring procedures and the manner of arranging the data. Table 1 shows a typical summation sheet for a representative group of nine lenses. The prescribed spherical and cylindrical powers of the given lens head the column. These values are converted to nominal meridional powers $V_{P}$ and $V_{H}$ whose difference is the nominal cylindrical power $C_{P}$. The axial meridional powers $V_{O}$ and $H_{O}$ are then measured in the proper manner and their difference $C_{o}$ is the measured axial cylindrical power. The marginal 
powers at $A$ and $B$ are then measured. It is customary to make measurements of the marginal meridional powers on both sides of the axis. For example, the final accepted measured value of one of the marginal meridional powers is the average obtained for the two equivalent areas symmetrically located on either side of the axial point of the spectacle lens. The measured powers for these equivalent areas should actually be the same but there are usually small differences which arise in part from errors of measurement and in part from actual differences in performance. The reliability of the final accepted values of marginal power at $A$ and $B$ is increased by this averaging process.

TABLE 1. Typical summation sheet for a group of nine lenses

The table shows the prescribed powers, Sph and $\mathrm{Cyl}$; the converted meridional prescribed powers, $V_{P}, H_{P}$, and $C_{P}$; the measured axial powers, $V o, H_{0}$, and the measured values of the deviations $\triangle V, \triangle H$, and $\triangle C$ at the points $O, A$, and $B$. All values are expressed in diopters.

\begin{tabular}{|c|c|c|c|c|c|c|c|c|c|}
\hline & \multicolumn{9}{|c|}{ Lens number- } \\
\hline & 1 & 2 & 3 & 4 & 5 & 6 & 7 & 8 & 9 \\
\hline Sph_. & 4. 00 & 4.00 & 4. 00 & 2.00 & 2.00 & 2. 00 & -2.00 & -2.00 & -2.00 \\
\hline $\mathrm{Cyl}$ & 2.00 & 1.00 & 0.00 & 2.00 & 1. 00 & 0.00 & 200 & 1. 00 & 0.00 \\
\hline $\mathrm{V}_{\mathrm{P}}$ & 6.00 & 5.00 & 4. 00 & 4.00 & 3.00 & 2. 00 & 0.00 & -1.00 & -2.00 \\
\hline $\mathrm{HP}_{\mathrm{P}}$ & 4.00 & 4.00 & 4. 00 & 2. 00 & 2. 00 & 2. 00 & -2.00 & -2.00 & -2.00 \\
\hline $\mathrm{C}_{\mathrm{P} \ldots}$ & 2.00 & 1.00 & 0.00 & 2.00 & 1.00 & 0.00 & 2.00 & 1. 00 & 0.00 \\
\hline Vo & 6.12 & 5.00 & 3.99 & 3. 98 & 3.01 & 2. 02 & -0.03 & -0.94 & -2.01 \\
\hline $\mathrm{Ho}_{\mathrm{o}}$ & 4.02 & 3.97 & 3.99 & 2. 01 & 2.04 & 2. 01 & -1.97 & -1.95 & -2.02 \\
\hline $\mathrm{C}_{0}$ & 2. 10 & 1. 03 & 0.00 & 1. 97 & 0.97 & 0.01 & 1.94 & 1.01 & 0.01 \\
\hline $\mathrm{V}_{\mathrm{A}}$ & 5. 97 & 4.87 & 3.87 & 3.87 & 3.05 & 1. 90 & 0.19 & -0.78 & -1.99 \\
\hline $\mathrm{H}_{\mathrm{A}}^{\mathrm{A}}$ & 3. 71 & 3. 82 & 3.85 & 1.78 & 2. 05 & 1. 92 & -1.96 & -1.88 & -1.94 \\
\hline $\mathrm{C}_{\mathrm{A}}$ & 2. 26 & 1.05 & 0.02 & 2. 09 & 1.00 & -0.02 & 2.15 & 1. 10 & -0.05 \\
\hline $\mathrm{V}_{\mathrm{B}}$ & 5. 80 & 4.81 & 3.85 & 4.00 & 3.04 & 1. 92 & -0.10 & -0.92 & -1.93 \\
\hline $\mathrm{H}_{\mathrm{B}}$ & 3.60 & 3. 70 & 3.86 & 1.90 & 2.01 & 1. 93 & -2.01 & -1.89 & -1.98 \\
\hline $\mathrm{C}_{\mathrm{B}}$ & 2. 20 & 1.11 & -0.01 & 2. 10 & 1.03 & -0.01 & 1.91 & 0.97 & 0.05 \\
\hline$\triangle V_{0}$ & 0.12 & 0.00 & -.01 & -0.02 & 0.01 & .02 & -0.03 & .06 & -.01 \\
\hline$\triangle \mathrm{H}_{\mathrm{O}}$ & .02 & -.03 & -.01 & .01 & .04 & .01 & .03 & .05 & -.02 \\
\hline$\triangle \mathrm{C}_{0}$ & .10 & .03 & .00 & -.03 & -.03 & .01 & -.06 & .01 & .01 \\
\hline$\triangle V$ & -.15 & -.13 & -.12 & -.11 & .04 & -.12 & .22 & .16 & \\
\hline$\triangle \mathrm{H}_{\mathrm{A}}^{\mathrm{A}}$ & -.31 & -.15 & -.14 & -.23 & .01 & -.09 & .01 & .07 & .08 \\
\hline$\triangle \mathrm{C}_{\mathrm{A}}$ & .16 & .02 & .02 & .12 & .03 & -.03 & .21 & .09 & -.06 \\
\hline$\triangle V_{B}$ & -.32 & -.19 & -.14 & .02 & .03 & -.10 & -.07 & .02 & .08 \\
\hline$\triangle \mathrm{H}_{\mathrm{B}}$ & -.42 & -.27 & -.13 & -.11 & -.03 & -.08 & -.04 & .06 & .04 \\
\hline$\triangle \mathrm{C}_{\mathrm{B}}$ & .10 & .08 & -.01 & .13 & .06 & -.02 & -.03 & -.04 & .04 \\
\hline
\end{tabular}

In making the measurements, it is customary to make and record five separate settings in the determination of each meridional power for each area. The average value obtained from these five settings is used in the determination of the power as measured by a single observer. The determinations of the meridional power are usually accurate to \pm 0.03 diopters.

Direct measurements are made only on the meridional powers. The values of cylindrical power are given by the difference in the two meridional powers for a given area. Consequently the probable error of a determination of the value of cylindrical power for a given area is usually greater than the probable error of determination of either of the meridional powers. The departures from the prescribed values of the various powers are shown in the lower part of table 1. It is clear that the magnitude of the variations from the prescribed powers is greater for the marginal area, $A$ and $B$, than for the central axial area.

At the time measurement of the marginal powers of spectacle lenses with respect to the vertex sphere by means of a modified vertex power measuring instrument was initiated, there was some uncertainty regarding the reliability of this method. Accordingly the marginal powers at $30^{\circ}$ off axis of a number of spectacle lenses were measured on, both the modified instrument and on the precision spectacle tester. Comparison of results obtained by the two methods showed good agreement. Table 2 shows a comparison of measured results for two lenses by the two methods. It is evident that the differences obtained for the two methods are well within the limits of error and can be neglected. It can therefore be

TABLE 2. Comparison of values obtained by two methods for two representative spectacle lenses

The nominal prescribed power for each lens is Sph 4.00, Cyl 1.00 diopter Column A gives the results for the modified vertex power measuring instrument and column B gives the results obtained with the spectacle tester. All values in the table are given in diopters.

\begin{tabular}{|c|c|c|c|c|c|c|}
\hline Lens No. & \multicolumn{3}{|c|}{1} & \multicolumn{3}{|c|}{2} \\
\hline Power & A & B & $\mathrm{B}-\mathrm{A}$ & A & B & $\mathrm{B}-\mathrm{A}$ \\
\hline $\begin{array}{l}V_{0} \\
\mathrm{Ho}_{-} \\
\mathrm{C}_{\mathrm{O}}\end{array}$ & $\begin{array}{l}\text { 5. } 03 \\
4.00 \\
1.03\end{array}$ & $\begin{array}{l}\text { 5. } 06 \\
4.00 \\
\text { 1. } 06\end{array}$ & $\begin{array}{r}0.03 \\
.00 \\
.03\end{array}$ & $\begin{array}{l}\text { 5. } 15 \\
4.10 \\
1.05\end{array}$ & $\begin{array}{l}5.15 \\
4.11 \\
1.04\end{array}$ & $\begin{array}{r}0.00 \\
.01 \\
-.01\end{array}$ \\
\hline $\begin{array}{l}V_{A} \\
H_{A} \\
C_{A}\end{array}$ & $\begin{array}{l}4.92 \\
3.90 \\
1.02\end{array}$ & $\begin{array}{l}\text { 4. } 93 \\
\text { 3. } 88 \\
\text { 1. } 05\end{array}$ & $\begin{array}{r}.01 \\
-.02 \\
.03\end{array}$ & $\begin{array}{l}\text { 5. } 06 \\
\text { 4. } 04 \\
\text { 1. } 02\end{array}$ & $\begin{array}{l}\text { 5. } 06 \\
\text { 4. } 05 \\
\text { 1. } 01\end{array}$ & $\begin{array}{r}.00 \\
.01 \\
-.01\end{array}$ \\
\hline $\begin{array}{l}V_{B} \ldots \\
H_{B} \\
C_{B} \ldots\end{array}$ & $\begin{array}{l}4.86 \\
3.76 \\
1.10\end{array}$ & $\begin{array}{l}\text { 4. } 84 \\
3.74 \\
1.10\end{array}$ & $\begin{array}{r}-.02 \\
-.02 \\
.00\end{array}$ & $\begin{array}{l}5.12 \\
3.86 \\
1.26\end{array}$ & $\begin{array}{l}5.09 \\
3.87 \\
1.22\end{array}$ & $\begin{array}{r}-.03 \\
.01 \\
-.04\end{array}$ \\
\hline
\end{tabular}

stated that, with the auxiliary modifying devices of the type described in this paper, the usual commercial type of vertex power measuring instrument can be used for the accurate measurement of the marginal meridional powers of a spectacle with respect to the vertex sphere.

Measurements on approximately 200 lenses have been made in the course of this research. Only a few of these measurements are reported here. The author expresses his appreciation to the four observers who have worked on this project. They are Walter R. Darling, Paul V. Barrans, Nancy Greenwood, and Loyd E. Sutton.

Washington, March 4, 1955. 